\title{
International Particle Physics Masterclasses - Current development to expand scope and global reach
}

\author{
Farid Ould-Saada ${ }^{1, *}$ \\ ${ }^{1}$ University of Oslo, Norway
}

\begin{abstract}
The International Particle Physics Outreach Group (IPPOG) is a network of scientists, science educators and communication specialists working across the globe in informal science education and outreach for particle physics. IPPOG's flagship activity is the International Particle Physics Masterclass programme, which provides secondary students with access to particle physics data using dedicated visualisation and analysis software. Students meet scientists, learn about particle physics, accelerators and detectors, perform physics measurements and search for new phenomena, then compare results in an end-of-day videoconference with other classes. The most recent of these events was held from 7 March to 16 April 2019 with thousands of students participating in 332 classes held in 239 institutes from 54 countries around the world. We report on the evolution of Masterclasses in recent years, in both physics and computing scope, as well as in global reach.
\end{abstract}

\section{Introduction - IPPOG and IMC}

The International Particle Physics Outreach Group (IPPOG) [1] is a network of scientists, science educators and communication specialists working across the globe in informal science education and outreach for particle physics. The International Masterclasses (IMC) [2], IPPOG's flagship activity, has developed an educational activity that brings the excitement of cutting-edge particle physics research into the classroom. The IMC programme on Particle Physics provides secondary students with access to particle physics data using dedicated visualisation and analysis software. During one day at a university, students meet scientists, learn about particle physics, accelerators and detectors, perform physics measurements and search for new phenomena, then combine and compare results in an endof-day videoconference with other classes. The LHC data have been successfully deployed since 2010 [3] where students perform various measurements based on proton-proton, leadlead and proton-lead collisions. The ambition to bring to the "classrooms" important LHC discoveries is realised using the discovery of the Higgs boson in 2012. Since then, the IMC programme has been extended beyond the LHC.

\footnotetext{
* Farid Ould-Saada: farido@uio.no, on behalf of the IPPOG collaboration
} 
We report on the evolution of Masterclasses in recent years, in both physics and computing scope, as well as in global reach.

\section{The 2019 International Masterclasses}

In the 2019 version of the IMC, held March 7 to April 16, over 10,000 high school students participated in 332 masterclasses at 239 institutions in 54 countries. The students made measurements of authentic data from four Large Hadron Collider (LHC) experiments at CERN and one experiment at Fermilab. The Belle II experiment at KEK was included in 2020. Moderation of videoconferences was shared by CERN, Fermilab, TRIUMF, and KEK, with the largest number of these at CERN (69) and Fermilab (27). Figure 1 shows two maps of countries who participated in the 2019 IMC in Europe and other continents.
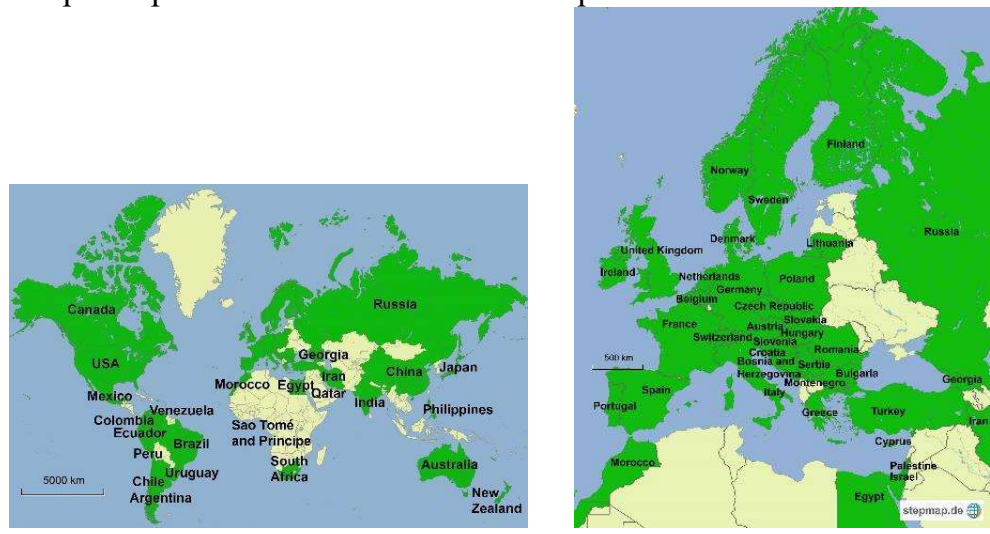

Figure 1. Countries involved in IMC 2019 from Europe (right) and other continents (left).

Compared to the previous years (

Figure 2), the attendance continues to increase, demonstrating that the IMC continue to be very popular among high school students and teachers. Ten new institutes joined in 2019: U. of M'Sila, Algeria, Kaunas U. of Technology, Lithuania, Tomsk Polytechnic U., Russia, U. of Sheffield, UK, UNAH, Tegucigalpa, Honduras, KazNU, Almaty, Kazakhstan, EPFL, Lausanne, Switzerland, MISIS, Moscow, Russia, U. of Sarajevo, Bosnia and Herzegovina, U. of Montenegro, Podgorica.

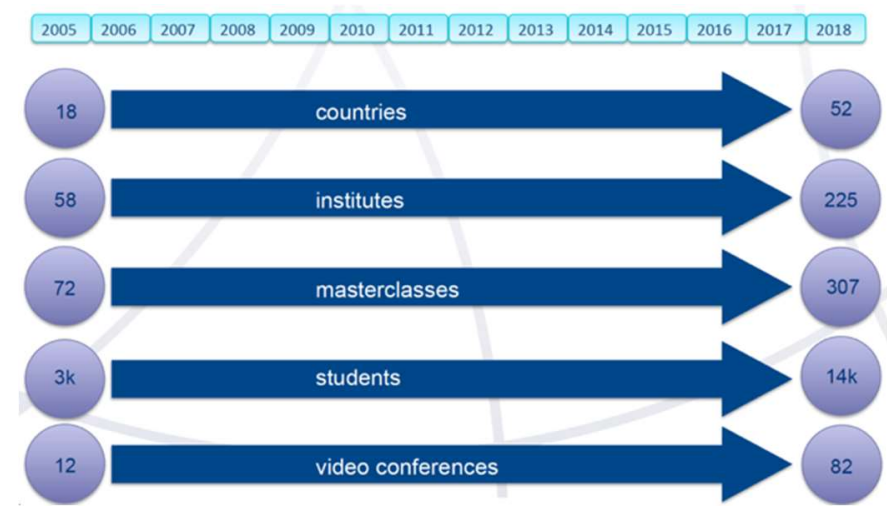

Figure 2. Statistics - International Masterclasses 


\section{Towards a multipath of International Masterclasses}

The LHC experiments steadily update their measurements or introduce new ones. New masterclass measurements beyond those for the LHC have been developed and tested. Some non-LHC, non-CERN groups began to develop masterclasses, notably IceCube and Pierre Auger. Belle II (KEK) physicists began work on the Belle II masterclass. GSI Darmstadt and the Heidelberg Ion Therapy Centre began to pioneer a particle therapy masterclass [4]. QuarkNet and Fermilab collaborated to develop and roll out accelerator-based neutrino masterclasses with the ultimate goal of producing a masterclass for the Deep Underground Neutrino Experiment (DUNE). The MINERvA neutrino masterclass is the first IMC offering in neutrino physics and the first based on a Fermilab experiment. More neutrino masterclasses are in development as well, especially for MicroBooNE.

\subsection{CMS masterclass updates}

In the "WZH" measurement of CMS, students look for leptonic decays to make ratios of $\mathrm{W}^{+}$ to $\mathrm{W}^{-}$candidates as well as of muons to electrons. They also make a mass plot which shows apparent $\mathrm{Z}, \mathrm{J} / \Psi$, and $\mathrm{Y}$ peaks. Students can also add a very limited number of Higgs candidates. In 2018, nearly 300 four-lepton events became available, showing apparent peaks for $Z \gamma$ and $\mathrm{ZZ}$ events as well as a smaller sample close to the Higgs mass. Work is underway to incorporate the new data so that students can make meaningful two- and four-lepton mass plots (Figure 3) from their masterclass analysis.

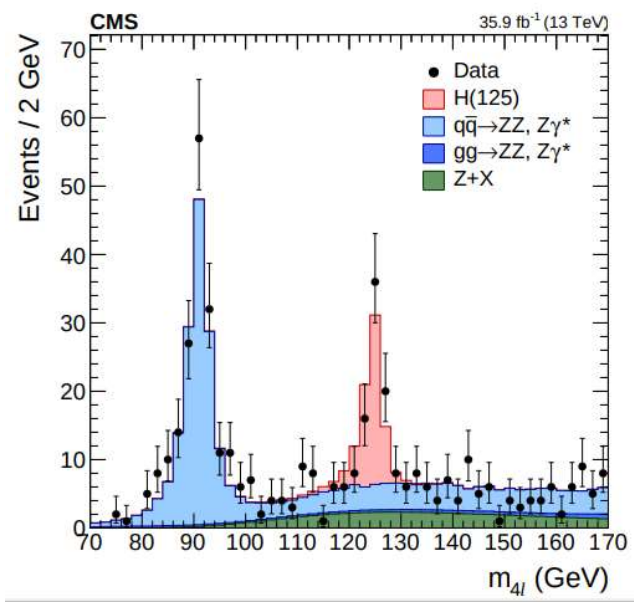

Figure 3. Four-lepton invariant mass from CMS showing, in addition to the Z-boson at $91 \mathrm{GeV}$, a clear indication of the Higgs boson at $125 \mathrm{GeV}$.

\subsection{ATLAS masterclass future plans}

The ATLAS ZPATH [5] education material is being extended to feature data events with dileptons and missing transverse energy, as well as Monte Carlo samples with corresponding supersymmetry and dark matter signals (Figure 4). As longer-term project the 13-TeV Open Data and Tools [6] will be deployed for high school students at the IMC (notebooks in Python, use of Virtual Machines, improved visualisation, machine learning in addition to standard analysis). 

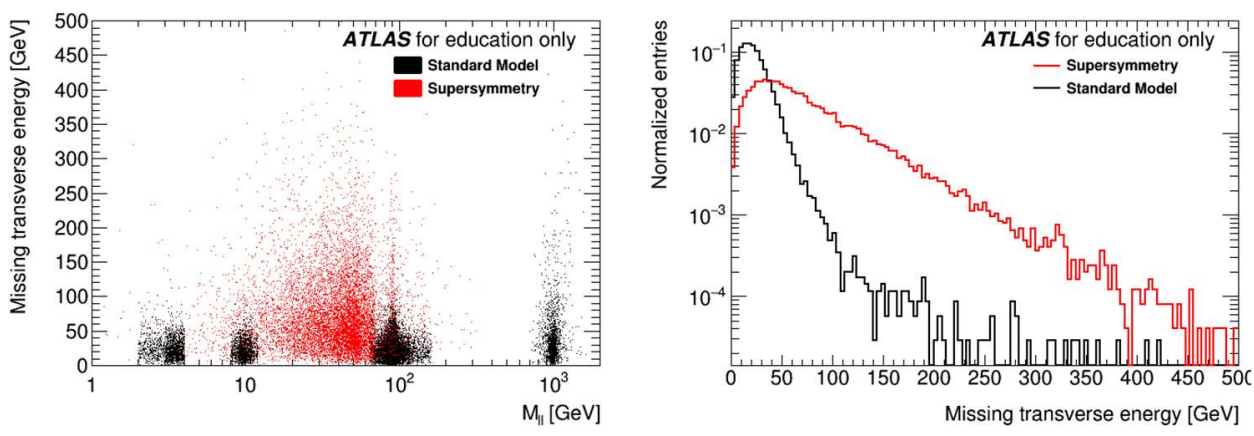

Figure 4 - Distributions of di-lepton invariant mass and missing transverse energy: ATLAS data for education and corresponding SM and Beyond SM Monte Carlo signals.

\subsection{Belle II masterclasses}

Belle II is an upgraded version of the Belle experiment at KEK to study B physics coming from electron-positron collisions. Based on the highly successful KEK B-Lab for students, the Belle II masterclass concentrates on large numbers of collision events. The students make use of a code made available to them in order to filter data and make a variety of plots, such as the invariant mass of 2 photons. The Belle II masterclass [7] was piloted in the 2019 IMC, improved and then permanently included in IMC 2020.

\subsection{Particle therapy masterclasses}

Medical physics has a great potential to capture the interest of students who are more oriented toward life sciences or are more motivated by applications of science to society. Student participants examine cancer scenarios and plan treatment with photon, carbon nucleus, and proton therapies, calculating optimal doses using the open source matRad toolkit. The particle therapy masterclass [8] will be introduced into International Masterclasses in 2020.

\subsection{Neutrino Masterclasses}

As Fermilab and other institutions have begun to push the intensity frontier with acceleratorbased neutrino experiments, physicists and educators became more interested in masterclasses to bring this physics to teachers and students. In 2018 and 2019, QuarkNet, Rochester physicists, and Fermilab developed a full masterclass, which debuted and was tested in International Masterclasses 2019, using conservation of momentum to draw conclusions. In the MINERvA measurement [11], students are able to study interactions of a neutrino beam with carbon nuclei. They examine events in the MINERvA detector (Figure 5), first separating signal from background and then using conservation of momentum and the uncertainty principle to test models of the interaction. The more successful model enables students to find the neutrino beam energy, Fermi motion of neutrons in the nucleus, and an approximation of the carbon nuclear radius. Student success at the measurement and interest were both high. 


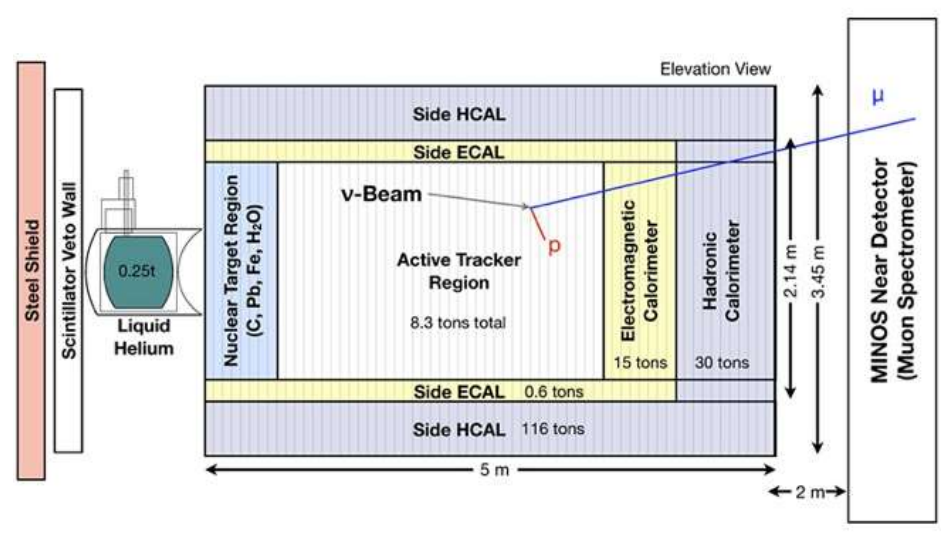

Figure 5. MINERvA Neutrino detector at Fermilab.

Fermilab MicroBooNE physicists have been collaborating with QuarkNet to develop a detector commissioning masterclass. The liquid argon time-projection chamber (TPC) technology in MicroBooNE is sophisticated and sensitive; students can examine cosmic ray muon tracks in the detector to determine the purity of the argon and the overall response of the detector. The MicroBooNE masterclass will be tested in IMC 2020. These activities have spurred interest from other neutrino experiments so that several more are expected to come online in the incoming years. DUNE (Deep Underground Neutrino Experiment) physicists are already considering a simulated supernova measurement based on the projected capabilities of the detector.

\section{Other extensions of the IMC Masterclasses}

The IMC have grown in a number of ways. World Wide Data Day (W2D2) and special masterclasses for International Day of Women and Girls in Science (IDWGS) are innovations that began two years ago and have taken root. W2D2 establishes new ways for high school students and teachers to engage in masterclass activities from their own classrooms. For IDWGS, a new pathway has been opened for high school girls to be encouraged in physics.

\subsection{World Wide Data Day - W2D2}

The purpose of the World Wide Data Day (W2D2), a single day of videoconferences, is to have a compact masterclass-style experience performed completely from school, without the need to visit a masterclass institution or even involve a physicist locally. Teachers help students to analyse data from ATLAS and CMS and look specifically for dimuon events. These events are easy to spot, even without making any adjustments to the event display. Students then measure the direction angles $\theta$ and $\phi$ of each muon by setting the display to the $z-y$ and $x-y$ views of the event, respectively, and using a protractor. ATLAS and CMS use a right-handed coordinate system with its origin at the nominal interaction point (IP) in the centre of the detector and the $\mathrm{z}$-axis along the beam pipe. The $\mathrm{x}$-axis points from the IP to the centre of the LHC ring, and the y-axis points upwards. In the transverse plane, the polar angle $\theta$ is between the direction of the particle and the z-axis and the azimuthal angle $\phi$ is around the z-axis. Students bin the results into $20^{\circ}$ intervals on a tally sheet; the class uses the tally sheets to make class histograms of $\theta$ and $\phi$ and to enter their results into a Google spreadsheet which displays 
histograms showing the combined results worldwide. The real physics discussion takes place in the W2D2 videoconference [9]. W2D2 was piloted in December 2016; it was held again in November 2017, 2018 and 2019. In 2018, over 1000 students at 69 institutions, mostly schools, in 19 countries, participated. Forty schools took part in 2019.

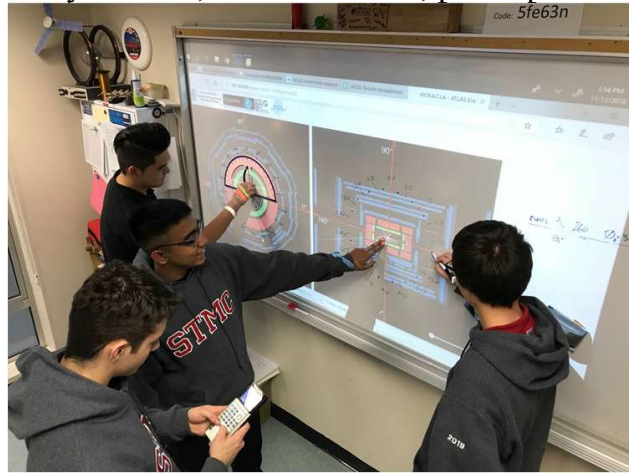

Figure 6. World Wide Data Day, Burnaby, Canada 2018: ATLAS Measurement.

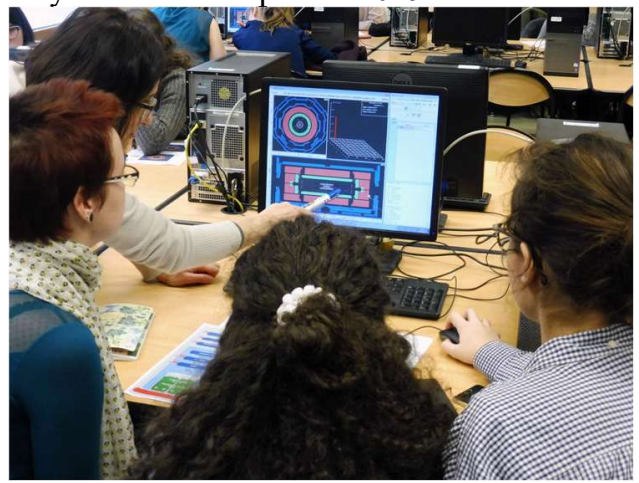

Figure 7. International Day of Women and Girls in Science.

\subsection{International Day of Women and Girls in Science - IDWGS}

Since 2016, February 11 each year has been the United Nations International Day of Women and Girls in Science (IDWGS) [10]. On and around that day each year, special masterclasses for women and girls have been held, united by CERN-moderated videoconferences which focus not only on the physics, but also on issues and opportunities for women in science. The IMC effort for IDWGS emphasizes masterclasses in a friendly physics environment for young women and female physicists as role models for the students. Most of the participants are high school girls, and women are well represented among the masterclass tutors and organizers. The moderators at CERN are women. In 2018 (2019), there were 12 IDWGS masterclasses in five (six) European countries plus Brazil. This increased to 17 masterclasses in 9 countries (7 in Europe, Brazil, South Africa) in 2020.

\section{Conclusions and outlook}

The particle physics International Masterclasses have been very successful and keep growing. A 3D-expansion is in place reaching more students and teachers, and more countries, and involving more activities and fields. The era towards a multi-"Path" of Masterclasses has started, expanding from particle physics at LHC to neutrino physics, Belle II physics, as well as to instrumentation and particle therapy. Some scalability challenges need be tackled both in terms of organisation and coordination, the growing number of centres for videoconferences.

Discussions have started at the November 2019 IPPOG meeting on how to expand and optimize the overall structure involving various fields, such as nuclear physics, astrophysics and cosmology, gravitational waves, and being part of international days, like the Worldwide Data Day and the International Day of Women and Girls in Science.

From the computing, software and data handling side, recent advances and developments, widely discussed during the 2019 CHEP conference, started to help minimise the overhead related to programming, software installation, data downloading, etc., such that more time is 
devoted to "research and measurements". With the advent of CERN Open Data and Tools, students acquire particle-physics knowledge by making Standard Model measurements, searching for new physics and reproducing as closely as possible important published results. The resources already deployed allow students to easily access and analyse data using desktops or laptops, practise programming (Python \& $\mathrm{C}++$ ) and adapt Jupyter notebooks using both Python and C++ ROOT to analyse data and Monte Carlo samples and produce physics analysis results using a Virtual Machine as a server. The students have thus the opportunity to work with modern software tools based on new technology and get an insight into principles of cloud and distributed computing. It is our intention to bring this into high schools. Another goal is to add new masterclasses in new Information Technology, computing, data science and machine learning technologies to the multipath of International Masterclasses.

\section{References}

[1] International Particle Physics Outreach Group: http://ippog.org/.

[2] International Masterclasses: http://physicsmasterclasses.org/.

[3] International Masterclasses in the LHC era, M. Bardeen, H.P. Beck, U. Below, K. Cecire, F. Ould-Saada, M. Kobel, CERN Courier, 22 May 2014, $\mathrm{http}: / /$ cerncourier.com/cws/article/cern/57305.

[4] New developments for ALICE Master Classes and the new Particle Therapy Master, L. K. Graczykowski, these CHEP 2019 proceedings.

[5] The ZPATH project, https://www.mn.uio.no/fysikk/english/research/projects/zpath/.

[6] ATLAS Open Data - Development of a simple-but-real HEP data analysis framework, F. Ould-Saada, these CHP 2019 proceedings.

[7] Belle II Masterclass https://belle2.ijs.si/public/.

[8] Particle Therapy Masterclass https://indico.cern.ch/event/840212/.

[9] World Wide Data Day website, https://quarknet.org/content/world-wide-data-day.

[10] United Nations General Assembly, Resolution adopted by the General Assembly on 22 December 2015, https://www.un.org/en/ga/search/view_doc.asp?symbol=A/RES/70/212.

[11] MINERvA Masterclasses https://indico.fnal.gov/event/22340/.

[12] Neutrino Masterclass Project Map 2019, https:/quarknet.org/content/neutrinomasterclass-project-map-2019. 